Documentation et bibliothèques

DOCUMENTATION BIBLIOTHËQUES

\title{
LA CRÉATION D’UN RÉSEAU QUÉBÉCOIS D’ACCÈS À LA DOCUMENTATION AYANT TRAIT AUX PERSONNES HANDICAPÉES
}

\section{Sophie Janik et Sylvie Laverdière}

Volume 33, numéro 2, avril-juin 1987

URI : https://id.erudit.org/iderudit/1052587ar

DOI : https://doi.org/10.7202/1052587ar

Aller au sommaire du numéro

Éditeur(s)

Association pour l'avancement des sciences et des techniques de la documentation (ASTED)

\section{ISSN}

0315-2340 (imprimé)

2291-8949 (numérique)

Découvrir la revue

Citer cet article

Janik, S. \& Laverdière, S. (1987). LA CRÉATION D’UN RÉSEAU QUÉBÉCOIS D'ACCÈS À LA DOCUMENTATION AYANT TRAIT AUX PERSONNES HANDICAPÉES. Documentation et bibliothèques, 33(2), 67-70.

https://doi.org/10.7202/1052587ar

Tous droits réservés ( $)$ Association pour l'avancement des sciences et des techniques de la documentation (ASTED), 1987
Ce document est protégé par la loi sur le droit d'auteur. L'utilisation des services d'Érudit (y compris la reproduction) est assujettie à sa politique d'utilisation que vous pouvez consulter en ligne.

https://apropos.erudit.org/fr/usagers/politique-dutilisation/ 


\section{LA CRÉATION D'UN RÉSEAU QUÉBÉCOIS D'ACCÈS À LA DOCUMENTATION AYANT TRAIT AUX PERSONNES HANDICAPÉES}

La création et l'organisation du réseau documentaire concernant les personnes handicapées est une initiative du centre de documentation de l'Office des personnes handicapées du Québec (OPHQ). Cette action s'inscrit d'ailleurs parfaitement dans le mandat principal de l'OPHQ qui est la coordination des services dispensés aux personnes handicapées du Québec. De façon plus spécifique, pour le centre de documentation, cela signifie la gestion et la diffusion de l'information relative à l'amélioration de la situation des personnes handicapées ${ }^{1}$.

D'autre part, pour certaines de ces activités, le centre de documentation de l'Office travaille déjà en quelque sorte en réseau. Ainsi, après avoir choisi et traité la documentation relative à l'amélioration de la situation des personnes handicapées, il la diffuse par les moyens appropriés à tous les intéressés et avant tout aux organismes de promotion et aux personnes handicapées. II reçoit également les informations documentaires (listes d'acquisitions, etc.) d'autres organismes s'il y a lieu. II répond aux besoins documentaires du milieu concerné et grâce à sa connaissance des ressources existantes, il guide l'usager dans ses recherches de l'information. II sert d'exemple en ce qui concerne la gestion de collection et fournit des conseils professionnels à ceux qui en font la demande. D'ailleurs de nombreux services documentaires ont déjà profité de son expertise dans ce domaine. Enfin, à moyen terme, il vise à mettre en place une banque de données bibliographique. Pour ce faire, il a élaboré sous forme d'un thésaurus ${ }^{2}$, un langage documentaire contrôlé qui sera la clé de l'organisation conceptuelle de ladite banque ${ }^{3}$.

Compte tenu de ce contexte, on comprendra alors qu'au lieu de créer un réseau, il s'agit plutôt d'officialiser et de consolider la collaboration entre les partenaires et de se doter d'une structure organisationnelle souple et ouverte. Les participants à ce réseau sont avant tout les associations de personnes handicapées que l'on appelle aussi organismes de promotion et un certain nombre de centres de réadaptation.

\section{Qu'est-ce qu'un réseau?}

Le réseau est une structure organisée pouvant assurer l'accès le plus simple, le plus rapide, le plus sûr et le plus économique possible à l'information dans le but d'assurer sur tous les points du territoire desservi et pour tous les citoyens, un accès universel aux publications ${ }^{4}$.

II ne faut pas que les expressions "réseau» et "partage de ressources" ressassent des clichés déjà vieux comme la gérance de la décroissance et le «faire plus avec moins». Le réseau, c'est l'établissement d'une communication, l'échange des informations, un partage d'idées et de ressources, l'augmentation de la productivité et l'amélioration des conditions de travail. L'important ce n'est pas tellement le réseau luimême, le produit fini, mais plutôt le procédé pour arriver à réaliser le réseau, les communications qui créent les liens entre les gens ${ }^{5}$.

\section{Le réseau et ses activités}

Pour créer le réseau il fallait d'abord s'assurer que les intervenants déjà engagés jugent utile et même nécessaire d'officialiser leur collaboration et de s'organiser en réseau. En janvier 1986, le centre de documentation de l'OPHQ a effectué un sondage téléphonique auprès de plusieurs centres de documentation spécialisés dont celui de l'Institut Raymond-Dewar. Cette consultation a révélé un intérêt des responsables des centres pour un tel projet et leur désir d'y collaborer.
1. "Loi assurant l'exercice des droits des personnes handicapées», Lois refondues du Québec, Chapitre E-20,1.

2. Sophie Janik et al., Thésaurus: Personne handicapée, Québec, ministère des Communications, 1986, 429 p.

3. Sophie Janik, «Thésaurus: Personne handicapée, outil de coordination des services documentaires spécialisés", Documentation et bibliothèques, Vol. 33, no 1 (janvier-mars 1987), $11-17$.
4. Roderick M. Duchesne, Quelques termes relatifs au réseau canadien de bibliothèques, Ottawa, Bibliothèque nationale du Canada, 1982.

5.John Naisbitt, Megatrends, ten directions transforming our lifes, New York, Varnes, 1982. 
Suite à cette démarche, le centre de documentation de l'Office a préparé un document de travail sur le sujet ${ }^{6}$. Celui-ci a été présenté au conseil d'administration de I'OPHO au début d'avril. Entre-temps, la Confédération des organismes provinciaux des personnes handicapées du Québec était informée du projet. Celui-ci y a d'ailleurs été très favorablement accueilli, $65 \%$ des membres de ce groupement ont manifesté leur intention de s'intégrer au réseau.

Les représentants d'une trentaine d'organismes de promotion et de centres de réadaptation assistèrent à la première réunion du réseau, le 29 avril 1986. Ils ont suggéré de faire d'abord un état de la situation sur les ressources de différents centres ou services documentaires, leur fonctionnement actuel, leurs besoins spécifiques, etc. Un questionnaire devant permettre de répondre en partie à ces questions fut distribué aux participants. Trois comités furent également formés pour approfondir des dossiers spécifiques d'intérêt commun à partir desquels on pourra établir et structurer les sphères d'activités.

\section{Comité circulation}

Les membres de ce comité doivent se pencher sur les processus et les problèmes de circulation de l'information surtout en ce qui concerne les organismes de promotion dont les ressources sont restreintes. Ils doivent aussi analyser les besoins des petits organismes qui ne prévoient pas pouvoir s'informatiser, du moins à moyen terme.

\section{Comité média substituts}

II est formé d'organismes engagés dans ce domaine. Les membres se sont fixés comme objectif de produire un catalogue des documents en média substituts qui concernent directement la problématique des personnes handicapées.

\section{Comité informatique}

Celui-ci a pour mandat de préparer un dossier sur les possibilités d'automatisation, les ressources actuelles de certains centres, les besoins des participants, les subventions possibles et les systèmes déjà existants. D'après le sondage effectué par l'un des membres du comité, les partenaires du réseau s'intéressent beaucoup à l'informatique et souhaiteraient avoir une journée d'information sur le sujet. On leur présenterait alors différents systèmes informatiques, tels Arctel, Infopuq, Access, Inet 2000, IST Informathèque, etc. Le comité a par ailleurs l'intention de préparer une bibliographie analytique des documents qui portent sur l'informatique au service des personnes handicapées.

Outre les décisions prises au sujet de la formation de comités, les participants à la réunion ont opté pour l'utilisation d'un langage documentaire commun, celui que propose le Thésaurus: personne handicapée?

Signalons aussi qu'à cette même réunion, des personnes ressources avaient été invitées à venir nous faire part de leur expérience. II s'agissait de deux représentants du réseau de l'Association des bibliothèques de santé affiliées à I'Université de Montréal (ABSAUM) et d'une représentante du réseau Accès et réintégration à la communauté via la télécommunication (ARCTEL). Ce système mis sur pied à l'Institut de réadaptation de Montréal utilise un babillard électronique bilingue. Celui-ci, équipé d'un modem, devient un moyen accessible de communication et d'interaction pour les personnes handicapées.

En dernier lieu, il faudrait souligner un trait spécifique de ce réseau. Mis à part quelques organismes comme l'Institut Nazareth et LouisBraille, le Centre Constance-Lethbridge et I'Institut Raymond-Dewar, où il existe des ressources professionnelles pour superviser le fonctionnement de bibliothèques déjà bien implantées, la plupart des membres du réseau ne disposent pas de services de spécialistes en documentation. II faut donc que le réseau mette l'emphase sur l'aspect du soutien mutuel et que les professionnels expérimentés puissent jouer un rôle de personnes ressources dans différentes activités bibliothéconomiques. Finalement, en guise de conclusion, nous aimerions faire nôtres les paroles de Gérard Mercure prononcées lors d'un congrès de I'ASTED: "La constitution d'un réseau n'est pas d'abord une affaire d'informatique ni d'interconnexion d'ordinateurs. C'est surtout une habitude de concertation, une volonté collective de réaliser des programmes communs»8.

\section{Sophie Janik}

Responsable du Centre de documentation Office des personnes handicapées

Drummondville

\section{Sylvie Laverdière}

Responsable du Centre de documentation Institut Raymond-Dewar

Montréal
6. Sophie Janik, Vers la création d'un réseau d'accès à la documentation ayant trait aux personnes handicapées, Drummondville, Office des personnes handicapées du Québec, 1986, $18 \mathrm{p}$.
7 Sophie Janik et al., "Thésaurus: personne handicapée..."

8. Gérard Mercure, Les réseaux québécois actuels: forces et faiblesses, présenté au Congrès de l'ASTED, Québec, 27 au 29 octobre 1982, 14 p. 


\section{BIBLIOTHĖQUES ET CENTRES DE DOCUMENTATION CONCERNANT LES PERSONNES HANDICAPÉES PAR TYPE DE DÉFICIENCE}

\section{- Autisme}

Société québécoise pour l'autisme, Montréal. (514) 931-2215

\section{Communautés culturelles}

Association multi-ethnique pour l'intégration des personnes handicapées du Québec,

Montréal

(514) 272-1573

\section{Déficience auditive}

Association du Québec pour enfants avec problèmes auditifs, Montréal

(514) 842-3926

Centre québécois de la déficience auditive, Montréal

(514) 845-3057

Institut Raymond-Dewar, Montréal

(514) 284-2581

\section{Déficience intellectuelle}

Association de Montréal pour les déficients mentaux, Montréal

(514) 381-2307

Institut québécois de la déficience mentale, Montréal

(514) 849-3616

\section{Déficience motrice}

Centre François-Charron, Québec

(418) 529-9141

\section{Déficience physique}

Centre de réadaptation Lucie-Bruneau, Montréal (514) 527-4521

Institut de réadaptation de Montréal, Montréal (514) 735-3741

\section{Déficience physique et mentale}

Centre de réadaptation Constance-Lethbridge, Montréal

(514) 487-1770

\section{Déficience visuelle}

Association montréalaise pour les aveugles,

Montréal

(514) 489-8201
Association québécoise des parents d'enfants handicapés visuels, Montréal

(514) 849-8729

Institut national canadien pour les aveugles, Montréal

(514) 284-2040

Institut Nazareth et Louis-Braille, Montréal (514) 463-1710

Regroupement des aveugles et amblyopes du Québec, Montréal

(514) 849-7515

\section{Dystrophie musculaire}

Association canadienne de la dystrophie musculaire, Bureau du Québec, Montréal (514) 861-3678

\section{Épilepsie}

Ligue de l'épilepsie du Québec, Montréal (514) 342-6877

\section{Grands brûlés}

Association québécoise pour le bien-être des brûlés, Montréal

(514) 845-9536

\section{Média substituts}

Institut national canadien pour les aveugles, Montréal

(514) 284-2040

Institut Nazareth et Louis-Braille, Montréal (514) 463-1710

Magnétothèque, Montréal

(514) 524-6831

Regroupement des aveugles et amblyopes du Québec, Montréal

(514) 849-7515

\section{Paraplégie}

Association des paraplégiques du Québec, Montréal

(514) 340-2053

Santé mentale

Auto-psy, Québec

(418) 529-1978 
Auto-psy, Montréal

(514) 694-0320

\section{Sclérose en plaques}

Société canadienne de la sclérose en plaques, Division du Québec, Montréal

(514) 849-7591

\section{Spina-bifida et hydrocéphalie}

Association de spina-bifida et d'hydrocéphalie du Québec,

Montréal

(514) 340-1075

\section{Tourisme}

Kéroul, Association de développement touristique pour les

personnes handicapées du Québec, Montréal (514) 252-3104
Troubles d'apprentissage

Association du Québec pour enfants et adultes ayant des troubles d'apprentissage, Montréal (514) 861-5518

\section{Généralités (toutes les déficiences)}

Office des personnes handicapées du Québec, Drummondville

(819) 477-7100

N.B.: Cette liste n'est pas exhaustive, elle ne signale que les organismes qui ont participé à la première réunion du réseau.

\section{Errata}

Une erreur s'est glissée dans la transcription de deux articles de la dernière livraison de la revue, les textes étant maintenant soumis sur disquettes. Nous nous en excusons auprès de nos lecteurs et collaborateurs. 